\title{
Arc-welding quality assurance by means of embedded fiber sensor and spectral processing combining feature selection and neural networks
}

\author{
J. Mirapeix* a , P.B. García-Allende a , A.Cobo ${ }^{a}$, O. Conde ${ }^{a}$, J.M. López-Higuera ${ }^{a}$ \\ ${ }^{a}$ Photonics Engineering Group, University of Cantabria, Avda. de los Castros S/N, 39005 Santander, \\ Spain
}

\begin{abstract}
A new spectral processing technique designed for its application in the on-line detection and classification of arc-welding defects is presented in this paper. A non-invasive fiber sensor embedded within a TIG torch collects the plasma radiation originated during the welding process. The spectral information is then processed by means of two consecutive stages. A compression algorithm is first applied to the data allowing real-time analysis. The selected spectral bands are then used to feed a classification algorithm, which will be demonstrated to provide an efficient weld defect detection and classification. The results obtained with the proposed technique are compared to a similar processing scheme presented in a previous paper, giving rise to an improvement in the performance of the monitoring system.
\end{abstract}

Keywords: fiber sensor, spectral processing, arc-welding, on-line monitoring

\section{INTRODUCTION}

Arc and laser welding on-line monitoring has been an active research topic in the last decades. The lack of knowledge regarding the physics involved in those processes, and their increasing relevance in several industrial niches have forced to look for reliable procedures to ensure the quality of the resulting welds. The use of non-destructive evaluation techniques (NDT) such as penetrant liquids, X-rays, magnetic particles or infrared thermography, is typically combined with procedure trials in test specimens or coupons, in an attempt of establishing optimal values for the input welding parameters (welding current, shielding gas flow rate, separation between the electrode tip and the seam). On-line monitoring systems have been proposed for both arc and laser welding [1]. In this regard, plasma optical spectroscopy is a promising alternative, as there exists a correlation between some plasma spectroscopic parameters, e.g. plasma electronic temperature, and the appearance of defects in the weld seams. In addition, on-line monitoring by means of real-time analysis is possible with this approach [2].

In terms of optical sensor system proposals, the particular characteristics of arc-welding have made it difficult to find a non-invasive solution. Input optics based on collimators attached to the welding torch have been proposed [3], but with limited applicability in general terms. In a previous communication we proposed a new sensor arrangement based on the use of an optical fiber embedded within a TIG torch [4]. In spite of the aggressive environment to be found in the vicinity of the plasma column, we demonstrate the feasibility of the proposed sensor system to be applied for online arc-welding quality monitoring.

When the traditional spectroscopic approach for welding quality monitoring is chosen, plasma electronic temperature profiles are obtained by using two or more atomic emission lines within those contributing to the plasma. Defect detection can be accomplished with this approach, but defect classification has not been reported. A new processing technique based on the use of PCA (Principal Component Analysis) as the dimensional reduction stage, and an ANN (Artificial Neural Network) designed and trained to perform the defect classification was proposed in a previous paper [5]. Although the validity of the technique was demonstrated, it gives rise to further investigations, mainly focused on providing information regarding the spectral bands selected by PCA to be used as the ANN inputs.

Some of the disadvantages to be found in the traditional feature extraction techniques such as Principal Component Analysis (PCA) or Linear Discriminant Analysis (LDA) are listed in the following [6]:

- When analyzing an unknown spectrum it is necessary to measure all the original features or spectral bands in order to perform the compression prior to its classification.

Third European Workshop on Optical Fibre Sensors, Antonello Cutolo, Brian Culshaw, José Miguel López-Higuera, Eds., Proceedings of SPIE Vol. 6619,66193P, (2007) $\cdot 0277-786 X / 07 / \$ 18 \cdot$ doi: $10.1117 / 12.738765$ 
- The interpretation of the results becomes a complex task given that the obtained features can not be associated with any of the spectral bands of the compounds under test.

- The obtained results can not be extended to other classifiers.

In this paper an algorithm based on feature selection, the Sequential Floating Forward Selection (SFFS) [7] is proposed to obtain the main features or spectral bands to be used afterwards in the classification stage of spectral data from an optical fiber-based sensor system. SFFS solves the disadvantages of PCA and LDA mentioned above, and in the particular case of plasma spectrum analysis, allows to establish an association between the selected bands and their corresponding emission lines found in the plasma spectra. A properly trained Artificial Neural Network (ANN) will be employed in the classification stage.

\section{DATA ANALYSIS}

\subsection{Sequential Floating Forward Selection}

Data compression by means of the SFFS algorithm is performed in two stages: redundancy reduction and spectral band selection.

1. Redundancy reduction: Spectral bands providing the same information to discriminate between correct welds and the different kind of defects have to be determined. A high correlation coefficient between two bands, nearly 1 , means that both offer the same information. Therefore, a data decorrelation is performed. First of all the correlation coefficient matrix is calculated and 51 blocks of correlated spectral bands, i.e. with a correlation coefficient higher than 0.99 , are obtained. Afterwards, a spectral band of each block, the most discriminant one according to the Bhattacharyya distance is selected. The Bhattacharyya probabilistic distance between two normal distributions is:

$$
J_{B}=\frac{1}{4}\left(\mu_{2}-\mu_{1}\right)^{T}\left[\Sigma_{1}+\Sigma_{2}\right]\left(\mu_{2}-\mu_{1}\right)+\frac{1}{2} \log \left(\frac{\left|\Sigma_{1}+\Sigma_{2}\right|}{2\left(\left|\Sigma_{1}\right| \cdot\left|\Sigma_{2}\right|\right) \frac{1}{2}}\right)
$$

where $\mu_{i}$ is the mean of the $i$ class; $\sum_{i}$ its covariance matrix and $|A|$ stands for the determinant of matrix $A$. In this case three different instabilities are discriminated from correct welds, therefore the criterion to select the most discriminant band must include the Bhattacharyya distance between every two distributions:

$$
J=\sum_{i=1}^{4} \sum_{j=i+1}^{4} P_{i} P_{j} J_{i j}
$$

where $P_{i}$ is the occurrence probability of correct welds and each of the three instabilities respectively.

2. Feature Selection: The problem of feature selection consists in, given a set of candidate features, selecting a subset with the best performance under some classification system. In this particular case the system performing the classification task is an Artificial Neural Network and the features are the different wavelengths of the acquired plasma spectra.

The SFFS algorithm begins with an empty feature subset. It iteratively adds and removes features until some termination criterion is fulfilled. This criterion is established by the user and it is the maximum number of features to be used. The term "forward" is used because it begins with an empty feature subset, and "floating" because after each forward step (addition of a new spectral band) some backward steps (removal of several spectral bands) are applied as long as the resulting subsets are better than the previously evaluated ones. The selection criterion is an objective function evaluated for each selected feature subset and it is a measurement of the separability of the classes. The probabilistic Bhattacharyya distance for a multiclass discrimination is again applied to measure this separability.

The spectral dimensionality reduction procedure is summarized in Figure 1. 


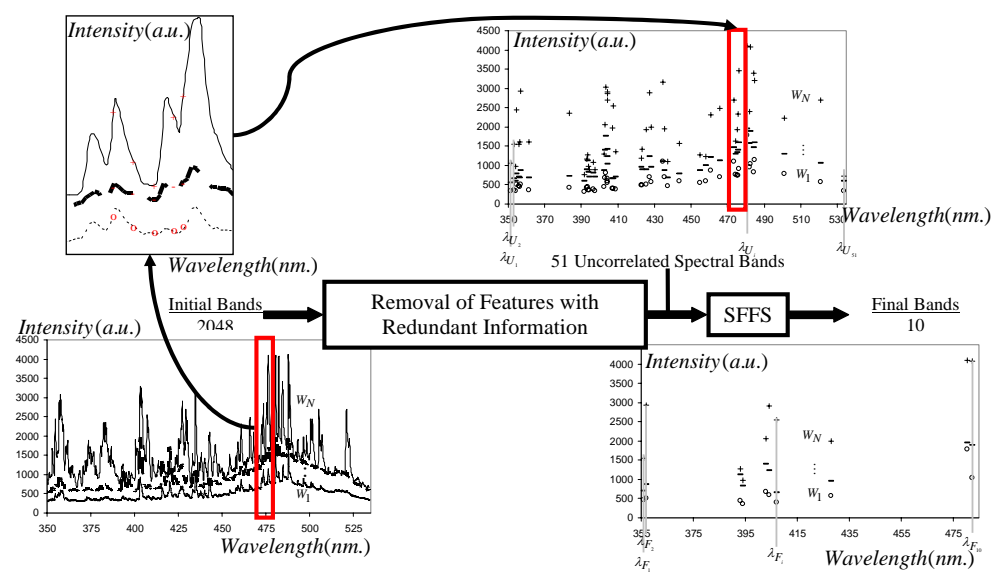

Fig. 1. Block diagram of the spectral dimensionality procedure.

\subsection{Classification}

The final selected bands are then classified using an artificial neural network [8-9]. The net is a multilayer feed-forward network, where a Back-propagation learning algorithm has been employed. It consists of an input layer and a single hidden layer, the two of them with 20 neurons or processing elements, and an output layer with only four neurons. The first neuron of the output layer is activated, which means that its output is nearly 1 , when the spectrum under test is classified as a correct weld and the second one if a reduction in gas flow is found. The activation of the third neuron indicates the presence of an incision (perturbation in the piece thickness) and finally the fourth neuron is activated in case of an insufficient welding current. The neurons in all layers have a log-sigmoid transfer function. This network topology is simpler than the one used in the work previously mentioned [6], then another additional advantage, a reduction in the mean classification time, is provided by the employment of SFFS instead of PCA in the compression stage. It is demonstrated in the following Section that this enhancement in the computational performance causes an inappreciable decrease in the system performance concerning the detection of instabilities.

\section{EXPERIMENTAL ISSUES}

Several welding tests were carried out to check the proposed technique. A TIG arc-welding system, composed of a Kemppi Mastertig 220 power supply and a Kemppi TIG torch TTC 220, where tungsten electrodes were employed (1 $\mathrm{mm}$ diameter) have been used for the welding tests. The specimens used in the experiments were stainless-steel AISI 304 plates attached to a positioning system (Newport MM4005 controller and MTM100PP1 positioning stage $(1 \mu \mathrm{m}$ precision)). Argon was selected as shielding gas, with a constant flow rate of $12 \mathrm{~L} / \mathrm{min}$. As mentioned in Section 1, an optical fiber (Ocean Optics P50-UVVIS) was used as a spectroscopic sensor embedded within the TIG torch [4]. The use of one of the gas nozzle exits to guide the fiber tip provides a cooling effect by means of the shielding gas. In addition, the shielding gas flow also prevents projections from the welding pool from damaging the fiber sensor. More than 200 welding tests have been carried out with the proposed optical sensor arrangement, enabling a complete spectroscopic analysis.

In Figure 2 the results corresponding to two weld experiments are shown. A defective weld seam is presented in Figure 2 (a), where a reduction in the welding current ( 50 to $44 \mathrm{~A}$ ) can be appreciated from $x \approx 3.5$ and $x \approx 5 \mathrm{~cm}$. It can be seen in Figure 2 (b) that the ANN output associated with the detection of low intensity is activated. The weld depicted in Figure 2 (c) presents the three possible defects: a reduction in the welding current (50 to $30 \mathrm{~A}$ ) from $x \approx 1.8$ to $x \approx 3.2 \mathrm{~cm}$, an incision from $x \approx 3.7$ to $x \approx 4.6 \mathrm{~cm}$ and a cut in the shielding gas flow from $x \approx 6$ to $x \approx 6.5 \mathrm{~cm}$. Figure 2 (d) shows the activation of the corresponding ANN outputs. 


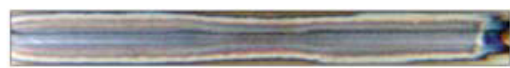

a) Seam with defects

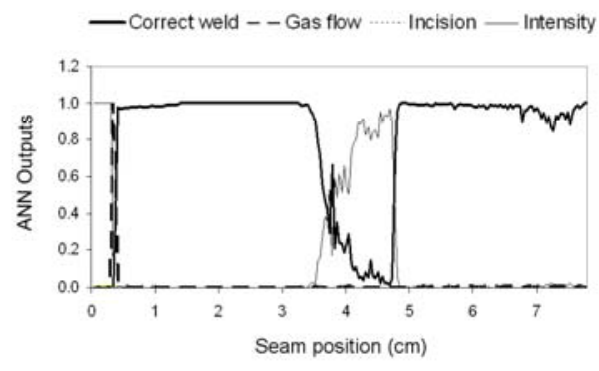

b) ANN outputs

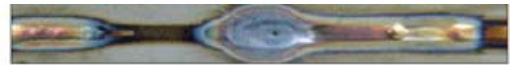

c) Seam with defects

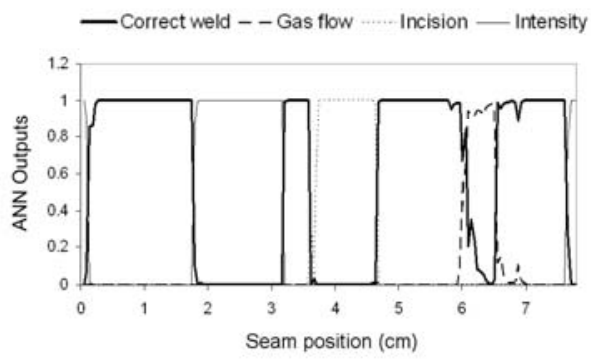

d) ANN outputs

Fig. 2. Example of classification results with defective welds.

\section{CONCLUSIONS}

A technique based on feature selection to reduce spectral dimensionality of data from an embedded fiber-sensor has been designed and successfully validated in the on-line detection and classification of arc-welding defects. Compared to feature extraction based-on methods, its main advantage lies in the identification of the physical meaning of the selected spectral bands. In addition, in this particular application, a reduction in the complexity of the classification algorithm has been achieved, what also implies an enhancement in the computational performance of the proposed technique.

\section{ACKNOWLEDGEMENTS}

This work has been co-supported by the Spanish TEC'2004-05936-C02-02 and TEC'2005-08218-C02-02 projects.

\section{REFERENCES}

1. M. Ferrara, A. Ancona, P.M. Lugara and M. Sibillano, "On-line quality monitoring of welding processes by means of plasma optical spectroscopy", Proceedings of SPIE 3888, 750-58 (2000).

2. J. Mirapeix, A. Cobo, O.M. Conde, C. Jaúregui and J.M. López-Higuera, "Fast algorithm for spectral processing with application to on-line welding quality assurance", Measurement Science \& Technology 17(10), 2623-9 (2006).

3. A. Ancona, P.M. Lugara, F. Ottonelli and I.M. Catalano, "A sensing torch for the on-line monitoring of the gas tungsten arc welding process of steel pipes", Meas. Sci. Tech. 15, 2412-18 (2004).

4. J. Mirapeix, A. Cobo, O.M. Conde, M.A. Quintela and J.M. López-Higuera, "Non-Invasive spectroscopic system for non-destructive arc-welding analysis", 9th ECNDT, P71, Berlin (2006).

5. J. Mirapeix, P.B. García Allende, A. Cobo, O.M. Conde and J.M. Higuera, "Real-time arc-welding defect detection and classification with Principal Component Analysis and Artificial Neural Networks", NDT\&E International, in print.

6. L. Gomez-Chova, J. Calpe, G. Camps-Valls, J.D. Martin, E. Soria, J. Vila, L. Alonso-Chorda and J. Moreno, "Feature selection of hyperspectral data through local correlation and SFFS for crop classification", IEEE International Geoscience and Remote Sensing Symposium. Proceedings (IEEE Cat. No.03CH37477) 1, 555-7 (2003).

7. Ferri F., Pudil P., Hatef M. and Kittler J., "Comparative study of techniques for large-scale feature selection". Pattern Recognition in Practice IV: Multiple Paradigms, Comparative Studies, and Hybrid Systems, E.S. Gelsema and L.N. Kanal, eds., North-Holland, Amsterdam, pp. 403-413 (1994).

8. C.H. Chen, Fuzzy Logic and Neural Networks Handbook, McGraw-Hill, New York, 1996.

9. F. Girosi, J. Makhoul, E. Manolakos and E. Wilson, Neural Networks for Signal Processing V, Proc. of the 1995 IEEE Workshop, IEEE Press, New York, 1995. 